\title{
Responsabilidad social corporativa en los agronegocios: dimensiones que influyen en el desempeño'
}

\author{
Corporate social responsibility in agribusiness: \\ dimensions affecting performance
}

\author{
Eva Martínez Caro² \\ Antonio Juan Briones Peñalver ${ }^{3}$ \\ Carmen de Nieves Nieto ${ }^{4}$
}

Fecha de recepción: 20 de enero del 2012

Fecha de aprobación: 19 de mayo del 2012

Trejos, E; Briones, A. Responsabilidad social corporativa en los agronegocios: dimensiones que influyen en el desempeño. Tecnología en Marcha. Vol. 25, Nº 6. Pág 23-28.

I Los datos de este trabajo proceden del Proyecto "Mejoramiento de las capacidades en los agronegocios de Costa Rica y la Región de Murcia, en las áreas emergentes de la economía, la responsabilidad social y las estrategias de cooperación de las empresas" financiado por el Programa de Cooperación Interuniversitario e Investigación Científica entre España e Iberoamérica, Agencia Española de Cooperación Internacional (AECID), Ministerio de Asuntos Exteriores y de Cooperación, Proyecto AECID (A/8/24/07).

2 Profesora, Universidad Politécnica de Cartagena, Departamento de Economía de Empresa, Escuela Técnica Superior de Ingeniería Industrial, España. Teléfono: (34) 968325775 Correo electrónico: eva.martinez@upct.es

3 Profesor, Universidad Politécnica de Cartagena, Departamento de Economía de Empresa, Facultad de Ciencias de la Empresa, España. Teléfono: (34) 968325943 Correo electrónico: aj.briones@upct.es

4 Profesora, Universidad Politécnica de Cartagena, Centro Universitario de la Defensa, España. Teléfono: (34) 968|8996। Correo electrónico: carmen.denieves@upct.es 


\section{Palabras clave}

Responsabilidad social, resultados, cultura, agronegocios.

\section{Resumen}

La Responsabilidad Social Corporativa (en adelante RSC) puede explicar el logro de ventajas competitivas. La RSC trata de minimizar los efectos negativos que ciertos aspectos de la organización provocan en el ambiente. Con el desarrollo de RSC, las empresas, además de llevar a cabo "buenas prácticas" en sus actividades, podrían ser consideradas agentes socialmente responsables y por consiguiente mejorarán su imagen, prestigio y reputación empresarial. La relación entre RSC y el rendimiento financiero ha sido objeto de un controvertido debate durante años. La presente investigación pretende aportar nuevo conocimiento sobre este debate planteándose como objetivo principal analizar cómo influye el fomento de la cultura en materia de RSC y, la contribución a la concreción de las dimensiones estratégicas o medidas de RSC, en los resultados de las empresas. Para ello se ha realizado un estudio empírico con una muestra de 226 empresas del sector agroindustrial. Los resultados indican que las dimensiones estratégicas con sus respectivos valores sociales como las medidas de RSC en las que se incluyen a los grupos de interés tienen influencia en los resultados empresariales.

\section{Key words}

Corporate Social Responsibility, business results, culture, agribusiness.

\begin{abstract}
Corporate Social Responsibility (hereinafter CSR) may explain the achievement of competitive advantages. CSR aims to minimize the negative effects that several aspects of the organization cause in the environment. With the development of a CSR, businesses, in addition to carrying out "good practices" in their activities, could be considered socially responsible agents, improving its image, status and corporate reputation. The relationship between CSR and financial performance has been the subject of controversial discussion for years. This research aims to provide new insights into this debate considering as main objective to analyze how it influences the development of culture in terms of CSR and the contribution to the realization of the strategic dimensions or measures of CSR, on business performance. An empirical study with a sample of 226 companies in the agribusiness sector was developed. The results indicate that both the strategic dimensions that refer to the social values as CSR measures involving interest groups, influence business results.
\end{abstract}

\section{Introducción}

En las últimas décadas se ha fomentado la cultura en materia de la Responsabilidad Social Corporativa (en adelante RSC). Cada vez más se ha intentado hacer concienciar en las organizaciones para que sus actuaciones tengan repercusiones en la actividad económica, en la sociedad y más concretamente en el medioambiente.

La visión contemporánea de la RSC constituye un campo de investigación y reflexión que, en la terminología anglosajona, se denomina stakeholder theory of the firm o teoría de la empresa como centro de relaciones de los diferentes grupos de interés y de los agentes empresariales. En este contexto, la RSC trata de minimizar los efectos negativos provocados en el ambiente por ciertos aspectos de la organización.

Las acciones tomadas en ese sentido están a favor de un desarrollo económico sustentable además de buscar la rentabilidad para los grupos de interés. De esta forma, las firmas además de llevar a cabo "buenas prácticas" en sus actividades, podrían ser consideradas agentes socialmente responsables, a la vez que están comprometidas con la sociedad (Bueno et al., 2004). Al mismo tiempo, las empresas pueden mejorar su propia imagen, prestigio y reputación empresarial, legitimando la organización frente sus actuaciones (Dias-Sardinha \& Reijnders, 200 I). 
La presente investigación tiene como objetivo principal analizar cómo influye el fomento de la cultura en materia de RSC así como analizar la contribución a la concreción de las dimensiones estratégicas o medidas de RSC en los resultados de las empresas. Para ello se ha realizado un estudio empírico con una muestra de 226 empresas del sector agroindustrial.

En el siguiente apartado se desarrollada el marco teórico de este artículo y se plantea la hipótesis principal. A continuación se detalla la metodología empleada, se muestran los análisis realizados y los resultados obtenidos. Finalmente se aportan las conclusiones del trabajo.

\section{Marco teórico}

la responsabilidad social de las empresas se puede definir como "la integración voluntaria, por parte de las empresas, de las preocupaciones sociales y medioambientales en sus operaciones comerciales y en sus relaciones con sus interlocutores (stakeholder)" (De Nieves \& Briones, 2009, p.89). Cuando se analizan las actividades económicas de las organizaciones, tanto aquellas producidas en el día a día operativo además las desarrolladas a partir de una adecuada planificación estratégica, como la entrada en nuevos proyectos y nuevos mercados, se observa que su objetivo es crear valor para los accionistas.

En este contexto, la puesta en práctica de medidas de RSC contribuyen a la buena reputación y prestigio de la empresa. Para lograr este fin, las "buenas prácticas" de RSC tratarán de satisfacer las expectativas de los grupos de interés (empleados, clientes, proveedores, accionistas, sociedad) además de contribuir con el desarrollo social, económicamente viable y ambientalmente sostenible.

\section{Valores de la cultura en RSC}

Kliksberg \& Tomassini (2000) plantean la hipótesis de que la introducción de la "dimensión cultural" y de la noción de "capital social" en el discurso referido al "desarrollo" constituye, en realidad, la búsqueda del objetivo crecimiento y la ampliación de la lógica del mercado a través de otros medios.

La toma de conciencia sobre las dimensiones culturales y los valores de la RSC en el desarrollo de los negocios surgen como un objetivo paralelo al crecimiento estratégico de las organizaciones. Las empresas que adoptan valores socialmente responsables, necesitan de una gestión estratégica (Vargas, 2004) la cual debe ser trazada en sus tres dimensiones: el desarrollo económico, la cohesión social y la protección del medioambiente (Jenkins \& Yakovleva, 2006).

Entonces, si la RSC afecta al modelo de gestión empresarial, existe la necesidad de alinear las medidas de dirección de las firmas con los valores organizacionales al generar mecanismos y herramientas capaces de impulsar cambios, sin dejar de lado la necesidad de adaptarse a las normas en esta materia (Silos, 2006, p.23). Con base en esto, los principales valores empresariales que contribuyen positivamente a una cultura de las empresas en materia de RSC son: I) el fomento del empleo y la inserción laboral, 2) la profesionalidad y la formación laboral, 3) la gestión responsable de la empresa y 4) la gestión medioambiental.

\section{Medidas para una dirección responsable}

La mayoría de empresas está adoptando iniciativas de RSC, que hasta ahora habían sido materia exclusiva de las grandes corporaciones (Díaz, 2005, p.29). Estos comportamientos organizacionales en materia de RSC son parte de la creación de valor de las firmas, y se ven incrementados con las prácticas del buen gobierno empresarial.

Uno de los retos de las empresas es poder comunicar los logros obtenidos por la gestión sostenible y la co-responsabilidad con los problemas sociales (Vargas, 2004) señalados en los informes, indicadores públicos de RSC y en las memorias de sostenibilidad.

Una política de responsabilidad corporativa bien desarrollada es un "activo invisible" (Imai \& Itami, 1984) el cual crea valor cuando está integrado en los sistemas operativos de una organización incorporando a los proveedores en el proceso de transformación. Al hacer esto se logra una mejora en la eficiencia con el objeto de atender las sugerencias y exigencias del cliente, conjuntamente con la implantación de políticas de mejora del entorno.

Por otro lado, la empresa puede buscar resultados en otras dimensiones no financieras como pueden ser el incremento de la motivación de los empleados, la 
adopción de un estilo de trabajo en equipo (Hayes, 2002), el desarrollo de acuerdos de cooperación (Gulati et al., 2000) y la creación de una cultura capaz de provocar un cambio organizativo, en aras de satisfacer a los clientes (Pettigrew y Fenton, 2000) cuyo fin es implantar políticas de mejora del entorno (Marín, 2008).

\section{Efecto de la RSC en el desempeño}

El objetivo de este trabajo es comprobar si las estrategias de RSC desarrollan capacidades empresariales que expliquen una mejora en el desempeño de las organizaciones. La relación RSC y el rendimiento financiero ha sido objeto de un controvertido debate desde los años sesenta como lo menciona De Quevedo (2003).

En opinión de Brío et al. (2005), las capacidades de las prácticas de RSC de las organizaciones explican los beneficios derivados de ellas en el logro de ventajas competitivas. Por otro lado, el capital social y la participación en redes sociales podrían llevar a reducir los costes económicos, así como a mejoras de dimensión y productividad (Castaño, 2007, p. 192).

Hart (1995) animó a las empresas a desarrollar recursos y capacidades en términos de RSC para conseguir beneficios a largo plazo asociados a una mayor rentabilidad para los socios. Por otro lado, la participación en la gestión o toma de decisiones democrática puede llevar a un reconocimiento de los empleados en las empresas que adoptan las prácticas de RSC.

El carácter voluntario de la RSC es enfatizado por los interlocutores sociales quienes consideran la necesidad de obtener una regulación internacional. Además, se destaca la relación con los derechos humanos y el impacto ambiental. Todos estos elementos pueden facilitar un mejor reconocimiento social de la empresa.

Estas apreciaciones fundamenta una hipótesis que relaciona las variables de la RSC que facilita el cumplimiento de los objetivos, con un reflejo en los posibles resultados de las empresas (dimensión, beneficio, productividad, reconocimiento, y rentabilidad): Existe una influencia significativa de los valores de la cultura en materia de RSC y de las medidas para una dirección responsable sobre el desempeño.

\section{Estudio empírico}

Población, muestra y recolección de información Los datos se obtuvieron por medio de una encuesta dirigida a 664 agronegocios de la Región de Murcia (España), entre los que se encontraban productores agrícolas, empresas dedicadas a la preparación y

Cuadro I. Constructos e ítems

\begin{tabular}{|c|c|}
\hline \multicolumn{1}{|c|}{ Variable } & Ítems \\
\hline Valores de la cultura & Fomento del empleo e inserción \\
\hline & Profesionalidad y formación laboral \\
\hline Medidas de RSC & Gestión responsable de la empresa \\
\hline & Gestión medioambiental \\
& Opiniones de los proveedores \\
\hline & Atender sugerencias de clientes \\
\hline Resultados de RSC & Políticas de mejora del entorno \\
\hline & Estilo de trabajo en equipo \\
\hline & Acuerdos de cooperación \\
\hline & Incremento del tamaño y la dimensión \\
\hline & Mejora de los resultados financieros \\
\hline & Incremento de la productividad \\
\hline & Reconocimiento de los empleados \\
\hline
\end{tabular}


conservación de frutas y hortalizas, venta de insumos, venta de equipos agrícolas, semillas y semilleros, diseño y asesoría del riego e invernaderos. Se obtuvieron 226 cuestionarios completos, de los cuales 84 se recibieron vía postal. Los 142 cuestionarios restantes, se obtuvieron mediante entrevistas personales. Las respuestas obtenidas, es decir un 34\% de la muestra total permiten realizar el estudio y establecer conclusiones.

\section{Indicadores y tratamiento de datos}

Tomando como referencia la revisión de literatura, se diseñó un cuestionario de quince ítems que miden los diferentes constructos de este estudio (tabla I). Todos los ítems se midieron a través de escalas Likert de cinco puntos.

Antes de realizar los análisis estadísticos pertinentes, es necesario asegurar que se están utilizando herramientas adecuadas para evaluar los conceptos que se pretenden medir. Por tanto, se comprobó la fiabilidad y validez de las escalas empleadas.

Para calcular la fiabilidad se utilizó el alfa de Cronbach, obteniéndose en todos los casos valores superiores a 0,7. La validez de cada escala se comprobó mediante un análisis factorial. Con este análisis se pretendió comprobar si cada escala estaba midiendo un único concepto. Antes de realizar el análisis factorial se determinó su conveniencia empleando el estadístico de Kaiser-Meyer-Olkin
(KMO) y el contraste de esfericidad de Barlett. Se obtuvieron valores satisfactorios en todos los casos.

El análisis factorial realizado muestra que dentro de la escala valores de la cultura en RSC subyacen dos factores. Por tanto, se definieron dos nuevas variables: valores sociales y valores ambientales. En las escalas medidas de RSC y resultados de RSC los análisis factoriales identificaron un único factor, confirmándose que dichas escalas estás midiendo un único concepto.

\section{Resultados}

Para contrastar la hipótesis planteada se realizó una regresión considerando como variable dependiente los resultados de RSC. Como variables independientes se tomaron los valores sociales (FVS), los valores ambientales (FVA) y las medidas de RSC (MRS). De los resultados se desprende la existencia de una asociación positiva de las variables independientes sobre la dependiente. Los valores culturales "sociales y ambientales" y las medidas de RSC explican el $41.8 \%$ de la varianza de los resultados en términos de RSC.

Se logró confirmar, parcialmente, la hipótesis, pues según se refleja en la tabla 2 no hay una relación significativa para el modo de gestión asociado a los valores ambientales. Sin embargo, la asociación es crítica para las dimensiones estratégicas cuyos valores sociales son aludidos, así como las medidas

Cuadro 2. Resultados del contraste de la hipótesis

\begin{tabular}{|c|c|c|c|c|c|c|}
\hline \multicolumn{7}{|c|}{ Desempeño } \\
\hline \multirow[t]{2}{*}{ Variables predictoras } & $\begin{array}{l}\text { Coeficientes } \\
\text { Estandarizados }\end{array}$ & $\begin{array}{l}\text { Prueba } T \\
\text { (Sig.) }\end{array}$ & \multicolumn{2}{|c|}{ Colinealidad } & \multicolumn{2}{|c|}{$\begin{array}{l}\text { Diagnóstico } \\
\text { Colinealidad }\end{array}$} \\
\hline & (Beta) & $(\mathrm{t})$ & $\mathrm{T}$ & FIV & A & IC \\
\hline (Constante) & & $3.632^{\mathrm{a}}$ & & & 3.903 & 1.000 \\
\hline$F_{1}:$ Valores sociales(FVS) & 0.314 & $\left.4.97\right|^{\mathrm{a}}$ & 0.750 & 1.334 & 0.050 & 8.876 \\
\hline$F_{2}$ VValores ambientales (FVA) & 0.022 & 0.317 & 0.607 & 1.648 & 0.031 & 11.229 \\
\hline $\mathrm{F}_{3}:$ Medidas de RSC (MRS) & 0.427 & $5.849^{a}$ & 0.559 & 1.789 & 0.017 & 15.188 \\
\hline $\begin{array}{l}R=0.654 ; R^{2}=0.427 \\
R \text { Ajustado }=0.418\end{array}$ & & \multicolumn{5}{|c|}{ Estadístico Durbin-Watson (DW) $=2.017$} \\
\hline Error estándar de la estimación $=2.1045$ & & \multicolumn{5}{|c|}{ ANOVA $(F)=47.75 \mathrm{I}^{\mathrm{a}}$} \\
\hline
\end{tabular}


de RSC que implican a los grupos de interés (agentes internos y externos). Estos componentes de la regresión, predicen efectos significativos y positivos en el desempeño empresarial.

\section{Conclusiones}

En este trabajo se ha buscado un acercamiento a determinadas dimensiones estratégicas de la dirección empresarial las cuales pueden facilitar el tan nombrado crecimiento sostenible. Estas medidas podrían mejorar los indicadores sociales y ambientales, las actuaciones hacia los grupos de interés, reportar un beneficio inherente a todos los resultados de las firmas y por lo tanto contar con un mejor desempeño empresarial.

Además, las estrategias de crecimiento con base en el capital social, la cultura empresarial, las medidas hacia los grupos de interés, son elementos clave de la sostenibilidad. En este sentido, las prácticas de RSC influyen de forma significativa cuando se trata de trabajadores y proveedores, con el establecimiento de equipos de trabajo y la incorporación de las opiniones de los proveedores, teniendo estas variables un efecto positivo y crítico sobre la dirección por valores.

\section{Bibliografía}

Brío, J. A.; Fernández, E. \& Junquera, B. (2005). Dificultad de imitación de las capacidades medioambientales y ventaja competitiva: un estudio empírico. En Revista Europea de Dirección y Economía de la Empresa, 14 (4), 59-80.

Bueno Campos, E. (coordinador). (2004). El gobierno de la Empresa. Madrid: Ediciones Pirámide.

Castaño, M. S. (2007). Los valores éticos del capital social. En Galindo, M.A. (coordinador): Ética y Economía. Madrid: Instituto de Estudios Fiscales.
De Quevedo, E. (2003). Reputación y creación de valor. Una relación circular. Madrid:Thompson.

De Nieves, C. \& Briones, A.J. (2009). Las empresas de la economía social y sus relaciones con las instituciones: colaboración con la universidad en asuntos medioambientales. CIRIECEspaña, Revista de Economía Pública Social y Cooperativa, (65), Agosto, 85- I I

Dias-Sardinha, I. \& Reijnders, L. (200I). Environmental performance evaluation and sustainability performance evaluation of organizations: an evolutionary framework. En EcoManagement and Auditing: 8 (2), 7 I-79.

Díaz, F. (2005), Gestión de la responsabilidad social corporativa. Revista de la Economía Social, Sociedad Cooperativa. I4, Marzo, $3^{\mathrm{a}}$ época.

Gulati, R.; Noria, N. \& Zaheer, A. (2000). Strategic networks. Strategic Management Journal. 21, 203-215.

Hart, S. L. (1995). A natural resource based view of the firm. Academy of Management Review, 20, 874-907.

Hayes, N. (2002). Dirección de equipos de trabajo. Madrid: Ed. Thomson.

Imai, K. \& Itami, H. (1984). Interpenetración of organization and market. International of Industrial Organitation. 2, 285-310.

Jenkins, H. \& Yakovleva, N. (2006). Corporate social responsability in the mining industry: exploring trends in social and environmental disclosure. Journal of Cleaner Production. 14, $27 \mid-284$.

Kliksberg, B. \& Tomassini, L. (2000). Capital social y cultura: claves estratégicas para el desarrollo. Buenos Aires: Banco Interamericano de Desarrollo. Fondo de Cultura Económica de Argentina. S.A.

Marín Calahorro, F. (2008). Responsabilidad social corporativa y comunicación. Madrid: Editorial Fragua.

Pettigrew, A.M. \& Fenton, E. M. (2000). Complexities and dualities in innovative forms of organizing. 279-300. En Pettigrew, A.M.; Fenton, E.M. (eds): The Innovating Organization. London: Sage Publications.

Silos, J. (2006). Herramientas para incorporar la RSE en la empresa: la norma SGE 2I, CIRIEC-España. Revista de la Economía Social, Sociedad Cooperativa. 27, Mayo, $3^{a}$ época.

Vargas, A. (2004). Empresas cooperativas, ventaja competitiva y tecnologías de la información. CIRIEC-España, Revista de Economía Pública, Social y Cooperativa. 49, 13 -29. 